\title{
Index of Biblical Texts ${ }^{1}$
}

Ac

1.15. 77

$1.16-17 \quad 76$

4.8-12 $83-84$

$8.20-23 \quad 84$

$13.16 \quad 76,77$

15.7-11 85

$17.24 \quad 195$

Apc

$3.20 \quad 408$

7.9408

10.1-2 446

$10.8-10 \quad 446,447$

$10.11 \quad 447$

$17.1 \quad 445$

$17.5 \quad 446$

19.11408

$20.4 \quad 408$

$21.3 \quad 408$

$21.8 \quad 399$

Col

$1.17 \quad 326$

1 Cor

$7.34 \quad 140$

2 Cor

$12.2 \quad 444$

$12.2-4 \quad 443$

$12.3 \quad 444$

$12.4 \quad 444$

Eccl.

$3.22 \quad 326$
Ex

$28.2 \quad 419$

$28.4 \quad 418-419$

$28.5 \quad 419$

28.17-20 419

28.34-35 419

Gn

1.1458

1.1-2 326

4.116

$4.10 \quad 196$

$\mathrm{Hbr}$

$11.14 \quad 196$

Jn

1.1-3 137

$2.11 \quad 46$

\begin{tabular}{ll}
$7.3 \quad 162$ \\
\hline .5
\end{tabular}

$\begin{array}{ll}7.5 & 162\end{array}$

\begin{tabular}{ll}
$7.6 \quad 163$ \\
\hline
\end{tabular}

$\begin{array}{ll}7.18 & 166\end{array}$

$\begin{array}{ll}7.23 & 167\end{array}$

$\begin{array}{ll}7.27 & 168\end{array}$

$7.34 \quad 169$

7.37-38 169

$7.42 \quad 168$

$8.12 \quad 176$

$9.32 \quad 199$

$10.21 \quad 164$

11.43-44 297

$11.44 \quad 296,297$

$12.47 \quad 408$

Lc
$7.16 \quad 137$
$8.55 \quad 277$
13.6-9 139
$18.35-43 \quad 102$

1 Only explicit quotations of texts are taken into consideration; the numbers correspond to the pages, they are in Italic if the quote is in the notes 


\begin{tabular}{|c|c|c|}
\hline $18.37-38$ & 91,91 & Prv \\
\hline $18.38-39$ & 96 & $8.22-25 \quad 327$ \\
\hline $22.14-46$ & 311 & $25.27 \quad 326$ \\
\hline 24.1459 & & \\
\hline Mc & & Ps \\
\hline 1.617 & & $32.1-2 \quad 230$ \\
\hline $\begin{array}{ll}1.0 & 17 \\
5.9 & 277\end{array}$ & & $101.4-5196$ \\
\hline $5.42 \quad 277$ & & $102.7 \quad 233$ \\
\hline $6.14 \quad 282$ & & $102.20-22 \quad 233$ \\
\hline 10.4691 & & \\
\hline $10.46-52$ & 102 & $1 \mathrm{Pt}$ \\
\hline $10.47 \quad 91$ & & $3.18-20 \quad 238$ \\
\hline $10.50 \quad 92$ & & $4.6 \quad 238$ \\
\hline $11.12-24$ & 144 & \\
\hline $14.17-42$ & 311 & Sap \\
\hline 16.2459 & & $9.16 \quad 326$ \\
\hline Mt & & $11.17 \quad 326$ \\
\hline $3.4 \quad 17$ & & II reg \\
\hline $3.10 \quad 139$ & & $5.7 \quad 130$ \\
\hline $8.13 \quad 278$ & & $66 \quad 465$ \\
\hline $\begin{array}{l}8.14-15 \\
8.15 \quad 278\end{array}$ & 48 & \\
\hline $9.8 \quad 137$ & & III reg \\
\hline 18.11408 & & $2.10 \quad 130$ \\
\hline $21.17 \quad 130$ & & \\
\hline $21.17-22$ & 144 & IV reg \\
\hline $25.33 \quad 521$ & -522 & \\
\hline $26.20-46$ & 311 & $2.24 \quad 278$ \\
\hline $28.1 \quad 459$ & & \\
\hline $28.18-19$ & 456 & $\mathbf{R m}$ \\
\hline $\mathrm{Ph}$. & & $3.23-24 \quad 408$ \\
\hline $3.20-21$ & 84 & $1 \mathrm{Tm}$ \\
\hline & & $6.17-19 \quad 82$ \\
\hline
\end{tabular}

